\title{
LA FORMACIÓN EN CONTABILIDAD DE GESTIÓN. ANÁLISIS DE LA OFERTA UNIVERSITARIA DE PREGRADO EN COLOMBIA, BRASIL Y ESPAÑA
}

\author{
Management accounting education. Analysis of the university offer in Colombia, \\ Brazil and Spain
}

\author{
Alina Marcela Bustamante-Salazar \\ Doctora en Administración, Universidad EAFIT. Medellín, Colombia. \\ alibs77@gmail.com
}

\section{Cómo citar / How to cite}

Bustamante-Salazar, A. (2016). La formación en contabilidad de gestión. Análisis de la oferta universitaria de pregrado en Colombia, Brasil y España. Revista CEA, 2(3), 13-30.

Recibido: 16 de septiembre de 2015

Aceptado: 12 de noviembre de 2015

\section{Resumen}

La contabilidad de gestión es un campo de conocimiento que tiene como finalidad apoyar a la gerencia en la toma de decisiones, a través de la planeación, el control de los recursos, la gestión y el análisis de información de costos; este es uno de los campos de actuación de los contadores. El propósito de este artículo es analizar la formación en Contabilidad de Gestión que se imparte en los programas de pregrado en Contaduría, Ciencias Contables, Finanzas y Contabilidad, que ofertan universidades de Colombia, Brasil y España. Para realizar el estudio fueron analizados los programas acreditados en alta calidad por el Ministerio de Educación Nacional de Colombia y los programas de pregrado ofertados por universidades brasileñas y españolas que figuran en el Ranking QS 2015 de Instituciones de Educación Superior de Latinoamérica y de Europa, respectivamente. Los resultados indican que la formación en Contabilidad de Gestión no ocupa un lugar significativo en los planes de estudio de programas de pregrado. Los temas de mayor interés están relacionados con los sistemas de costeo y análisis de costos tradicionales, el costeo y la gestión basados en actividades y herramientas de planeación y control gerencial.
Palabras clave: contabilidad de gestión, currículo de Contaduría, educación contable, programas de Contaduría.

\begin{abstract}
Management accounting is a field of knowledge which aims at supporting management in making decisions through the planning and control of resources, management and analysis of cost data; this is one of the areas of activity in accounting. The purpose of this article is to analyze the training in management accounting as taught in different undergraduate programs (Accounting, Accounting sciences, Finance and accounting) offered in Colombia, Brazil and Spain. To conduct the study high quality programs approved by the Ministry of National Education of Colombia were analyzed, as well as programs offered by Brazilian and Spanish universities listed in the QS University Ranking 2015 of Institutions of Higher Education in Latin America and Europe, respectively. The findings show that training in management accounting does not occupy a significant place in the curricula of undergraduate programs. The topics of greatest interest are related to costing systems and traditional cost analysis, activity-
\end{abstract}


based costing and activity-based management and planning tools and management control.

Keywords: management accounting, management accounting education, accounting curriculum, accounting education, accounting programs.

\section{INTRODUCCIÓN}

La contabilidad, concebida como sistema de información, está constituida por varios subsistemas que generan información que los grupos de interés -internos y externos- que las organizaciones utilizan para tomar decisiones. Uno de esos subsistemas es la contabilidad de gestión, campo de conocimiento que tiene como finalidad apoyar a la gerencia en la toma de decisiones, a través de la planeación y el control de los recursos, la gestión y el análisis de la información de costos. Esta se constituye en uno de los campos de formación y de actuación de los contadores.

En la década de 1980, la publicación del libro de Thomas Johnson y Robert Kaplan, Relevance Lost, traducido al español como La contabilidad de costes. Auge y caída de la contabilidad de gestión, suscitó la reflexión y generó cambios en el conocimiento y en las prácticas de contabilidad de gestión, que durante décadas estuvieron anquilosadas con técnicas y metodologías que habían sido diseñadas para las industrias de finales del siglo XIX e inicios del siglo $X X$, que resultaban impertinentes para el contexto en el que se desenvolvían las organizaciones, ad portas de un nuevo milenio. Del mismo modo, se cuestionó la formación en Contabilidad de Gestión que se impartía en los currículos de Contaduría.

Desde entonces, varios investigadores se han interesado en estudiar en este campo de formación para determinar su pertinencia, tal es el caso de Ahadiat (2008), Siegel, Sorensen, Klammer y Richtermeyer (2010), (Jiles, 2014) en el contexto estadounidense; João Lunkes, Ripoll
Feliu y Silva da Rosa (2012) en el español; y González (2010), en el contexto colombiano. Aunque se trata de entornos diferentes, estos estudios coinciden en argumentar que la contabilidad de gestión es un campo con gran potencial académico y profesional y en señalar la necesidad de fortalecer su formación.

Del mismo modo, otros autores han estudiado el desarrollo y las tendencias de investigación en contabilidad de gestión en el contexto iberoamericano. Al respecto, las investigaciones de Salgado-Castillo (2011), João Lunkes, Ripoll Feliu y Silva da Rosa (2013) concluyen que España y Brasil son los países iberoamericanos con mayor desarrollo en este campo, mientras el estudio de Duque Roldán y Osorio Agudelo (2013) señala que en Colombia esta investigación se encuentra en un estado incipiente.

Partiendo de los antecedentes enunciados anteriormente, esta investigación tiene como propósito comparar la formación en Contabilidad de Gestión que se imparte en algunos pregrados de contaduría de Colombia, Brasil y España, mediante el análisis de los planes de estudio y los micro-currículos, identificando las asignaturas, los temas, los créditos obligatorios y optativos dedicados a esta área de formación. En total fueron analizados 21 programas de pregrado, siete (7) de cada país, utilizando como criterios de selección, la acreditación de alta calidad para los pregrados de Colombia, y que estuvieran en el Ranking QS 2015 de Instituciones de Educación Superior de Latinoamérica y de Europa, para los pregrados de Brasil y España, respectivamente.

El artículo presenta en primera instancia, el marco teórico en el cual se explica el desarrollo histórico de la contabilidad de gestión. A continuación, se presenta una síntesis de algunas investigaciones previas sobre la formación en Contabilidad de Gestión. Finalmente, se presentan los resultados alcanzados con el estudio. 


\section{MARCO TEÓRICO}

\section{La contabilidad de gestión}

La contabilidad es definida por Aguiar, Cadavid, Cardona, Carvalho, Jiménez y Upegui (1998, p. 55) como un «sistema de medición y comunicación de hechos económicos y sociales relacionados con un ente específico, que permite a los usuarios juicios informados y decisiones conducentes a la colocación óptima de recursos y al cumplimiento de los objetivos de una organización».

Las interrelaciones que realizan las organizaciones y que son objeto de medición y representación en la contabilidad son muy diversas, algunas de ellas dan cuenta de asuntos fiscales, relacionados con el recaudo y pago de tributos, operaciones de inversión y financiación, adquisición y uso de recursos para la producción de bienes y servicios, acciones de responsabilidad social en beneficio de la comunidad, entre otras.

Son múltiples los grupos que pueden tener interés en conocer y controlar el desempeño de las organizaciones y muy variadas sus necesidades de información. De ahí que la contabilidad está estructurada por diferentes «subsistemas informativos que las empresas (...) modelan, desarrollan y utilizan para la producción de información que permita el cumplimiento de los fines de los diferentes usuarios que en tal organización convergen, así como los fines de la propia organización» (Gómez, 2007, p. 248). Así, por ejemplo, la contabilidad financiera y la contabilidad tributaria son subsistemas contables orientados a suministrar información a los grupos de interés externo, como los inversionistas, el Estado y la comunidad. Mientras que el control de gestión, la contabilidad de costos y la contabilidad de gestión, son subsistemas contables que suministran información para los grupos de interés interno.

La contabilidad financiera genera información sobre las transacciones y los hechos económicos que realizan las organizaciones, susceptibles de medición y reconocimiento, de acuerdo con unos principios, normas o estándares de contabilidad aplicables en cada país, proporcionando información sobre la situación financiera, el resultado de las operaciones, los cambios patrimoniales, los flujos de efectivo de la organización, en forma integral. La contabilidad tributaria suministra información para dar cumplimiento a las obligaciones fiscales de las organizaciones, de acuerdo con los parámetros definidos por las autoridades gubernamentales.

El control de gestión permite la evaluación de la eficiencia y la eficacia en el uso de los recursos por las diferentes áreas de la organización, y suministra información para la implementación de la estrategia más eficaz (Vanegas, García y Marín, 2009), (Loaiza, Cárdenas y Peralta, 2014).

La contabilidad de costos es un subsistema encargado de medir y calcular los costos de producción de los productos o servicios que ofertan las empresas, valorar los inventarios, calcular el costo de ventas y la rentabilidad, proporcionando información para la elaboración de los estados financieros, según las normas o estándares contables vigentes en cada país (Duque-Roldán y Osorio-Agudelo, 2013, pp. 1126-1127).

La contabilidad de gestión es «un área de conocimiento que implica la asistencia en el proceso de toma de decisiones, la planeación, la implementación de sistemas de gestión del desempeño, y proveer habilidades relacionadas con los reportes financieros y el control, y la asistencia a los gerentes en la formulación e implementación de la estrategia organizacional» (João Lunkes, Ripoll y Silva da Rosa, 2013, p. 12). La contabilidad de costos y la contabilidad de gestión utilizan la información que suministran los sistemas de costos, sin embargo, el campo de actuación de esta última es más amplio, pues no se limita a los costos sino además a la planeación y el control para apoyar la toma de decisiones (Hesford, Lee y Van Der Stede, 2007), (Harris y Durden, 2012). 
En el contexto iberoamericano, el término 'contabilidad de gestión' es usado por autores como Torrecilla, Fernández, y Gutiérrez (1993), Amat Salas, Amat Salas, y Álvarez López (1996), Escobar Rodríguez y Cortijo Gallego (2012). Otros autores como Escobar Bolívar (1991), Romero Ceceña (1997), Frezatti, De Aguiar, y Guerreiro (2007) utilizan el término contabilidad gerencial. También se utiliza el término contabilidad administrativa por autores como Sinisterra y Polanco (2007), Ramírez Padilla (2013), Calleja Bernal y Calleja (2014). Estos conceptos han sido utilizados indistintamente para referirse al mismo asunto, aunque pueden identificarse algunas tendencias, por ejemplo, en España es usual el término 'contabilidad de gestión', en Brasil es más común utilizar el concepto 'contabilidad gerencial', en Colombia se han usado los tres conceptos, pero en los últimos años se ha dado preferencia al término 'contabilidad de gestión'.

Una situación que contribuye al uso de esta multiplicidad de términos es la dificultad en la disciplina administrativa para diferenciar los conceptos gestión, gerencia y administración, pues, en algunos casos estos son usados como sinónimos, tal y como lo señala Sanabria (2010). Por tal razón, es necesario hacer una precisión sobre estos conceptos. La gestión es transversal a la organización, se concibe como la acción de comprender e intervenir la organización en sus múltiples niveles, funciones, procesos, proyectos y subsistemas (Sanabria, 2010, p. 189). La gerencia se refiere al rol que tiene a su cargo articular los esfuerzos de los actores que participan en una organización para lograr los objetivos propuestos para esta (Sanabria, 2010, p. 176). La administración se refiere a un campo de conocimiento a partir del cual se ha ido desarrollando la profesión administrativa (Sanabria, 2010, p. 189). A tenor de lo que se acaba de explicar, se considera que el concepto más apropiado es 'contabilidad de gestión', pues este es un subsistema que atraviesa todas las áreas de la empresa, que provee información para la toma de decisiones, por todos los estamentos de la organización, junto con la gerencia (Caldera, Baujín, Ripoll Feliu y Vega, 2007, p. 15).

Los orígenes de la contabilidad de gestión se remontan al siglo XIX cuando algunas empresas de textiles, ferrocarriles y metalúrgicas estadounidenses comenzaron a desarrollar herramientas para medir la eficiencia del trabajo y el uso de los recursos (Johnson y Kaplan, 1988, p. 24). En esa época, la contabilidad financiera y la de gestión operaban de forma independiente; la primera se ocupaba de medir el resultado global de la empresa; y la segunda, apoyaba la gestión interna de esta.

La contabilidad de gestión tuvo un gran desarrollo en la centuria comprendida entre 1825-1925, posteriormente entró en un periodo de decadencia (Johnson y Kaplan, 1988, p. 30), pues en la elaboración de los informes contables se dio prevalencia a las normas de contabilidad financiera que tenían como propósito medir el excedente económico global que generaban las organizaciones, y un menor interés en la generación de información para evaluar la eficiencia en el uso de los recursos, la medición de los costos y la determinación del rendimiento de cada línea de productos (Osorio, 1991, p. 7). Algunas situaciones que contribuyeron a esta decadencia están relacionadas con la separación entre la propiedad y la gerencia de la empresa, esto implicó un mayor interés en la auditoría de estados financieros, como forma de control y de evaluación del desempeño gerencial (Evia, 2006, p. 156). Por otra parte, las limitaciones en las tecnologías de la información de la época hacían muy costoso el procesamiento de los datos de la contabilidad de gestión, desestimulando su aplicación (Johnson y Kaplan, 1988, p. 129). Entonces, aunque en el periodo 1925-1980, desde la academia se propusieron algunos desarrollos -por ejemplo, los modelos cuantitativos aplicados a los costos-, estos tuvieron poca trascendencia en las prácticas de la época (Salgado-Castillo, 2014, pp. 793-794).

No obstante, el renacer de la contabilidad de gestión comienza a gestarse desde la década de 1980 con el surgimiento de nuevas 
metodologías, como los sistemas de costeo basado en actividades - Activity Based-Costing, $A B C$ - , la gestión basada en actividades Activity Based-Management, ABM-, el presupuesto basado en actividades - activity based-budgeting, $A B B-$, la gerencia estratégica de costos - Strategic Cost Management, SCM-, la teoría de las restricciones - Theory of Constraints, TOC - , el cuadro de mando integral -Balanced Scorecard, BSC-, entre otros.

Los sistemas de costos $A B C$ son un sistema de gestión empresarial que cuantifica el costo de los recursos que se utilizan en las actividades y en los procesos organizacionales, y posteriormente los asigna a los productos, servicios u otros objetos de costos - por ejemplo, los clientes, los proveedores - según las necesidades y requerimientos de cada empresa. La información que suministra estos sistemas es usada para la gestión basada en actividades, metodología administrativa, que tiene como propósito analizar las actividades de la organización y buscar alternativas para mejorar la eficiencia e incrementar la rentabilidad. Los presupuestos basados en actividades permiten determinar los recursos que requieren las áreas de la organización, basándose en las demandas previstas de actividades.

La gerencia estratégica de costos consiste en el conocimiento de la estructura de costos de una organización para desarrollar estrategias que permitan alcanzar ventajas competitivas duraderas (Shank y Govindarajan, 1998, p. 5), lo cual implica comprender la cadena de valor, el posicionamiento estratégico y las causales de costos. Por otra parte, la teoría de restricciones es un modelo de mejora continua, enfocado en identificar los puntos débiles o recursos escasos del sistema organizacional que limitan la generación de valor, para lo cual se requiere aumentar las utilidades, disminuyendo al mismo tiempo los inventarios y los gastos de operación. El cuadro de mando integral es un sistema de medición del desempeño basado en indicadores financieros y no financieros, que evalúa cuatro perspectivas organizacionales: la financiera, del cliente, los procesos internos y el aprendizaje y crecimiento; esto permite un monitoreo permanente y el establecimiento de planes de mejoramiento para alcanzar los objetivos propuestos.

Estos desarrollos de la contabilidad de gestión, parten de una comprensión sistémica de la organización, en la que ésta se estructura por procesos, además están vinculados a la estrategia organizacional. Se trata de metodologías para analizar e intervenir de un modo diferente las problemáticas organizacionales (Moehrle et al., 2009, p. 439).

\section{La formación en Contabilidad de Gestión}

Los procesos de globalización han aumentado los requerimientos de competitividad que deben afrontar las empresas, de ahí entonces que la contabilidad de gestión cobre una mayor importancia. Por esta razón, los currículos de Contaduría deben garantizar la formación de profesionales, con conocimientos y competencias pertinentes y actitudes éticas que les permitan desenvolverse en ese entorno competitivo, como gestores de los sistemas de información contable que proveen información para los grupos de interés internos y externos de las organizaciones. Tradicionalmente, tanto en la práctica como en la academia, se ha dado mayor énfasis a la contabilidad financiera y tributaria, dejando de lado otros campos de actuación como la contabilidad de gestión.

No obstante, tal como lo argumenta González (2010, p. 142): «la contabilidad de gestión ha venido ganando importancia en el mundo de los negocios desde que los gerentes se han dado cuenta que no es suficiente contar con la información que se encuentra en los estados financieros tradicionales, se requieren otros datos de carácter no financiero y del uso de herramientas gerenciales que en su conjunto faciliten el proceso de toma de decisión». Autores como Ahadiat (2008), Siegel et al. (2010), González (2010), Jiles (2014) consideran 
necesario fortalecer la esta formación en los currículos de los programas de Contaduría.

Al respecto, Ahadiat (2008) realizó un estudio entre contadores afiliados al Instituto de Contabilidad de Gestión - Institute of Management Accounting, IMA- y al Instituto Americano de Contadores Públicos Certificados -American Institute of Certified Public Accountants, AICPA- para identificar los conocimientos y las habilidades que las compañías esperan de los profesionales que se desempeñan en contabilidad de gestión en Norteamérica. Los resultados indican que la formación ética es clave en la formación de los contadores, así como el manejo de herramientas informáticas para la gestión de información, la elaboración de presupuestos operativos y de capital, la asignación de costos y los análisis de costo volumen utilidad. El autor concluye que los contadores deben desarrollar habilidades estratégicas y organizacionales, y que en los currículos de programas de contaduría deberían incluirse temas como la evaluación de la productividad y el desempeño, pues estos permiten medir la eficiencia con la cual se utilizan los recursos de la organización y la efectividad en el logro de los objetivos propuestos. Del mismo modo, es importante el estudio de la gestión del capital de trabajo, este se constituye en un concepto vital para el funcionamiento, la evaluación del riesgo y el rendimiento de las organizaciones. Así como incorporar la incertidumbre como parte de los procesos de planeación y fortalecer la formación en el desarrollo de sistemas de costos.

De otro lado, Siegel et al. (2010) sintetizan los resultados de varios estudios sobre la pertinencia de la formación en Contabilidad de Gestión en Estados Unidos, indagando entre los empresarios y los contadores con experiencia profesional. Los autores indican que es necesario fortalecer las competencias de los contadores en comunicación oral y escrita, negociación, trabajo en equipo y solución de problemas, e incluir en los currículos teorías y modelos de gestión de operaciones, mejoramiento de procesos, planeación estratégica, cadena de valor y logística, uso de tecnologías de la información y gestión de riesgos, así como herramientas para la planeación, el análisis de costos, la toma de decisiones y la evaluación de desempeño. Estas competencias y conocimientos le permitirán al contador comprender el contexto organizacional donde se desenvuelve y ofrecer los servicios profesionales que las empresas necesitan.

A su vez, la investigación de González (2010) sobre la enseñanza de la contabilidad de gestión en Colombia, identifica los desafíos que deben tenerse en cuenta para que los temas que se enseñan y las metodologías utilizadas estén acordes con la realidad empresarial. Al respecto, la autora indica que es necesario promover la participación en las asociaciones de contadores en los aspectos que afectan la profesión, repensar los temas y las metodologías que se usan en estos cursos y actualizar los microcurrículos para incluir temas como la gestión de la calidad total, justo a tiempo, cuadro de mando integral, costos basados en el tiempo invertido por actividad, teoría de las restricciones y toma de decisiones.

Del mismo modo, señala González (2010), es necesario que los estudiantes estén formados en temas de calidad, productividad y minimización de costos, que inciden en los factores de éxito de las organizaciones. Asimismo, la enseñanza de la Contabilidad de Gestión debe estar contextualizada a la estructura actual de los negocios, constituida principalmente por empresas de servicios que requieren metodologías de medición del desempeño y sistemas de costeo acordes con sus necesidades.

Para González (2010), el contexto de la investigación en contabilidad de gestión en Colombia, también debe ampliarse, pues esta está ligada a la estrategia de la empresa y la toma de decisiones. Entonces, es necesario desarrollar indicadores no financieros para medir los factores de éxito de los distintos tipos de organizaciones-públicas, privadas, comerciales, de servicios, entre otras - y utilizar metodologías cuantitativas para obtener 
estudios de mayor rigor. Finalmente, la autora considera que existe un nuevo campo de acción en el área que va mucho más allá de los sistemas de costeo y presupuestos tradicionales, ese nuevo campo de acción implica que los contadores se preparen para ser consultores empresariales o miembros de los grupos de gestión de las empresas. Para lograr este propósito se requiere que la formación en Contabilidad de Gestión sea acorde con el contexto en el que se desarrollan las organizaciones.

Precisamente, en el año 2014, el Instituto de Contabilidad de Gestión -Institute of Management Accounting, IMA- actualizó el contenido del examen para la certificación en Contabilidad de Gestión-Certified Management Accountant, CMA-, que se aplica a partir del año 2015. Este examen está constituido por dos partes: la primera parte evalúa las competencias relacionadas con informes financieros, planeación, desempeño y control; la segunda parte, hace énfasis en la toma de decisiones financieras. Según Jiles (2014), las temáticas del examen se constituyen en un referente para evaluar y actualizar los currículos de los programas de contaduría. El autor considera que es necesario profundizar en temas como gestión del desempeño, presupuestos y proyecciones, gestión de inversiones, control interno, análisis de decisiones, gestión del riesgo y ética profesional.

Todo lo anterior, evidencia la importancia de la formación en Contabilidad de Gestión como parte fundamental de los currículos de los pregrados en contaduría que se imparte en los diferentes países, en particular en Colombia, Brasil y España que son objeto de estudio en esta investigación, pues esta les permitirá a los contadores incursionar en un campo académico y de práctica amplio y tener una incidencia estratégica, como un profesional que agrega valor, en la gestión de las organizaciones.

\section{RESULTADOS Y DISCUSIÓN}

Esta investigación, de enfoque cualitativo y de carácter exploratorio, tiene como propósito comparar la formación en Contabilidad de Gestión que se imparte en los pregrados de Contaduría de Colombia, Brasil y España. Para realizar el estudio se tomaron, como referencia, los programas de pregrado en Contaduría Pública acreditados en Alta Calidad por el Ministerio de Educación Nacional de Colombia, según los datos del SNIES a noviembre de 2015; los programas de pregrado en Ciencias Contables de las universidades de Brasil, ubicadas en los 25 primeros lugares de Instituciones de Educación Superior latinoamericanas, según el ranking QS 2015, y los programas de pregrado en Finanzas y Contabilidad o Contabilidad y Finanzas, ofertados por universidades españolas mejor posicionadas en el ranking QS 2015 de Instituciones de Educación Superior de Europa. En total, se analizó la formación de pregrado en Contabilidad de Gestión de 21 programas, siete (7) de cada uno de los países seleccionados para el estudio, a través de un muestreo intencional, cuyos parámetros de selección estaban determinados por la calidad reconocida del pregrado - para el caso colombiano-, de la institución que lo oferta - para los pregrados de Brasil y España - y la disponibilidad del plan de estudios y de los microcurrículos, a través de las páginas de internet de las instituciones seleccionadas. La Tabla 1 indica las universidades de cada país incluidas en el estudio. 
Tabla 1. Universidades incluidas en el estudio

Table 1. List of Select Universities

\begin{tabular}{|l|l|}
\hline \multicolumn{1}{|c|}{ País } & \multicolumn{1}{|c|}{ Universidades } \\
\hline Colombia & $\begin{array}{l}\text { Pontificia Universidad Javeriana (Bogotá); Universidad del Valle; Universidad EAFIT; } \\
\text { Universidad Externado de Colombia; Universidad ICESI; Universidad Libre (Cali); Universidad } \\
\text { Santo Tomás (Bogotá) }\end{array}$ \\
\hline Brasil & $\begin{array}{l}\text { Universidad de San Pablo; Universidad Federal de Rio de Janeiro; Universidad de Brasilia; } \\
\text { Universidad Federal de Minas Gerais; Universidad Federal de Rio Grande del Sur; Universidad } \\
\text { Federal de Paraná; Universidad Federal de Santa Catarina }\end{array}$ \\
\hline España & $\begin{array}{l}\text { Universidad Autónoma de Barcelona; Universidad Carlos III de Madrid; Universidad de } \\
\text { Granada; Universidad de Zaragoza; Universidad de Sevilla; Universidad de Valencia; } \\
\text { Universidad de Alcalá }\end{array}$ \\
\hline
\end{tabular}

Fuente: elaboración propia

La posibilidad de acceder al microcurrículo de las asignaturas del área de contabilidad de gestión, fue una condición decisiva para la inclusión de los programas en el presente estudio. Para el caso colombiano, no se incluyeron, entre otros, los programas de Contaduría Pública ofertados por la Universidad de Antioquia, Universidad de Medellín y Universidad Militar Nueva Granada, pues, aunque tienen una trayectoria académica e investigativa en contabilidad de gestión, reconocida en el país, no tienen disponible al público el contenido detallado -microcurrículos o guías docentes - de las asignaturas objeto de estudio. A continuación, se presenta los resultados de los programas analizados.

\section{La Formación en Contabilidad de Gestión de algunos pregrados de Contaduría Pública de Colombia}

En Colombia, la Contaduría Pública es una profesión regulada, lo cual implica que solo pueden ejercer labores contables los graduados en Contaduría, que poseen una tarjeta profesional que los acredita para ejercer la profesión. En este país, la formación en Contaduría se caracteriza por la amplia oferta. Según los datos del Sistema Nacional de Información de la Educación Superior - SNIES, a noviembre de 2015 existen 121 Instituciones de Educación Superior que ofertan este pregrado, el $23,96 \%$ de estas entidades son públicas y el $76,04 \%$ son privadas; en total existen 238 programas de Contaduría con registro calificado activo, de los cuales el 10,92\% (26 programas) tiene certificación de alta calidad; estos se constituyen en la población objetivo de esta investigación y solo siete (7) de estos programas tienen publicados los microcurrículos de las asignaturas, estos fueron seleccionados en la muestra de esta investigación.

Al analizar la formación en Contabilidad de Gestión que reciben los estudiantes de Contaduría de los programas colombianos seleccionados en este estudio, se encuentra que en promedio el $8,28 \%$ de los créditos obligatorios del currículo se dedican a Contabilidad de Gestión, pocas universidades ofertan líneas de profundización u optativas en esta área de conocimiento, de los programas estudiados, se destacan la Universidad EAFIT y la Pontificia Universidad Javeriana (Bogotá), que ofrecen a los estudiantes la posibilidad de profundizar en esta área, dedicando el 9,38\% y el $7,50 \%$ de los créditos del plan de estudio, respectivamente, y la Universidad Libre que oferta la asignatura Gerencia Estratégica de Costos, que representa el 2,45\% de los créditos del currículo; así se muestra en la Figura 1.

Al indagar sobre el contenido de las asignaturas, se encuentra que en los cursos obligatorios se estudian principalmente los fundamentos, los sistemas y el análisis de costos, incluyendo aspectos básicos de presupuestos y proyecciones; algunos programas comienzan a incorporar la línea de toma de decisiones y simulación gerencial, como parte de la 
las restricciones. La Tabla 2 presenta las asignaturas obligatorias y optativas que ofertan los programas estudiados.

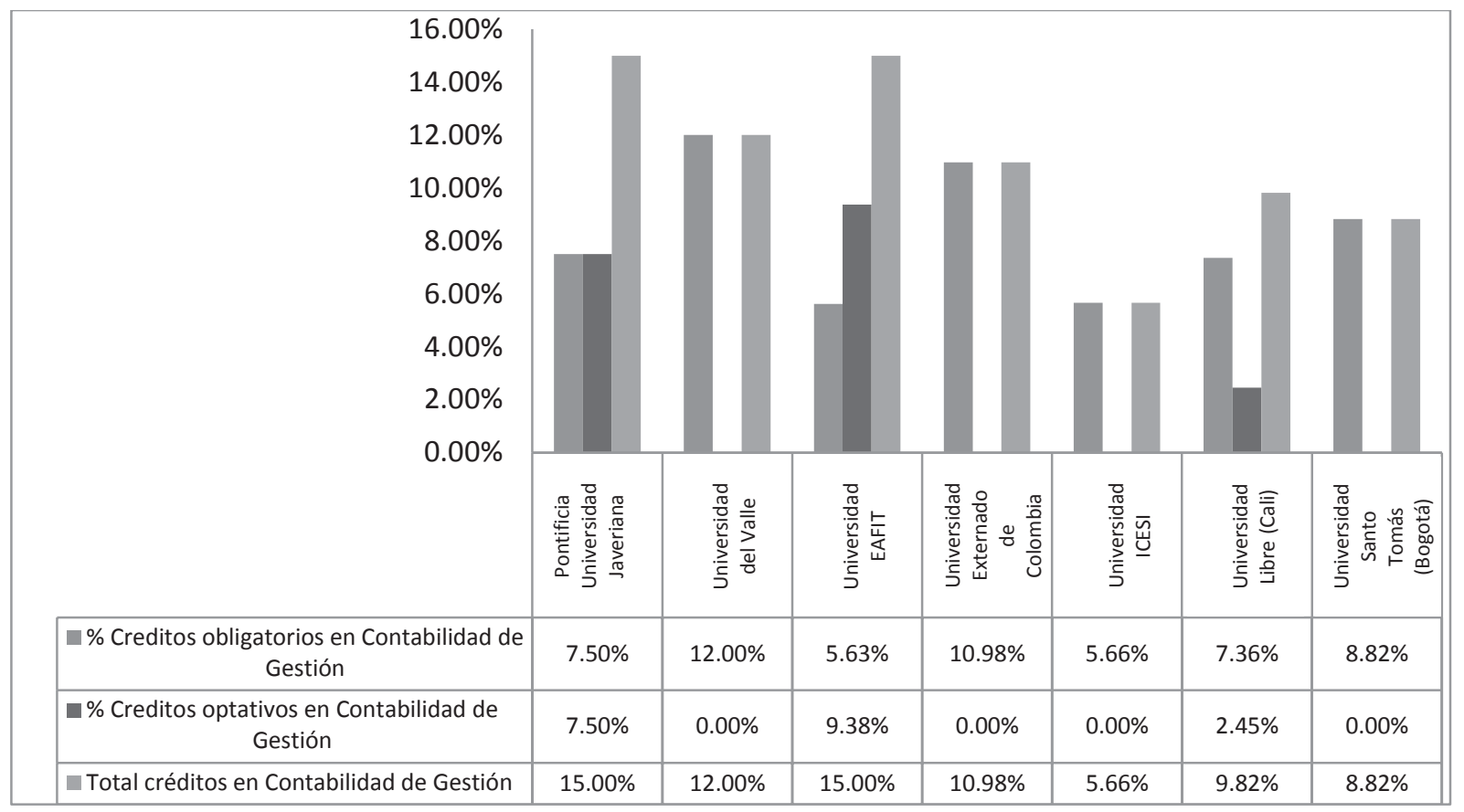

Figura 1. Porcentaje de créditos obligatorios y optativos dedicados a Contabilidad de Gestión en programas de Contaduría colombianos

Figure 1. Percentage of mandatory and electives credits dedicated to Management Accounting in Colombian Accounting Undergraduate Programs

Fuente: elaboración propia

Tabla 2. Asignaturas obligatorias y optativas en Contabilidad de Gestión de programas de contaduría colombianos.

Table 2. Mandatory and electives courses in Management Accounting in Colombian Accounting Undergraduate Programs.

\begin{tabular}{|l|l|l|l|}
\hline \multicolumn{1}{|c|}{ Institución } & \multicolumn{1}{|c|}{ Pregrado } & \multicolumn{1}{c|}{ Asignaturas Obligatorias } & \multicolumn{1}{c|}{$\begin{array}{c}\text { Asignaturas Optativas } \\
\text { (Electivas) }\end{array}$} \\
\hline $\begin{array}{l}\text { Pontificia } \\
\text { Universidad } \\
\text { Javeriana } \\
\text { (Bogotá) }\end{array}$ & $\begin{array}{l}\text { Contaduría } \\
\text { Pública }\end{array}$ & $\begin{array}{l}\text { Contabilidad de costos I; Contabilidad de } \\
\text { costos II; Contabilidad Gerencial I; } \\
\text { Contabilidad Gerencial II }\end{array}$ & $\begin{array}{l}\text { Gerencia Estratégica de } \\
\text { Costos I; Gerencia } \\
\text { Estratégica de Costos II; } \\
\text { Gerencia Estratégica de } \\
\text { Costos III }\end{array}$ \\
\hline $\begin{array}{l}\text { Universidad } \\
\text { del Valle }\end{array}$ & $\begin{array}{l}\text { Contaduría } \\
\text { Pública }\end{array}$ & $\begin{array}{l}\text { Análisis de Costos y Presupuestos; Control } \\
\text { de Gestión; Fundamentos de Costos; } \\
\text { Procesos de Costos I; Procesos de Costos } \\
\text { II; Resolución de Problemas de Toma } \\
\text { Decisiones; Sistemas de Producción }\end{array}$ & N/A \\
\hline
\end{tabular}




\begin{tabular}{|c|c|c|c|}
\hline Institución & Pregrado & Asignaturas Obligatorias & $\begin{array}{c}\text { Asignaturas Optativas } \\
\text { (Electivas) }\end{array}$ \\
\hline $\begin{array}{l}\text { Universidad } \\
\text { EAFIT }\end{array}$ & $\begin{array}{l}\text { Contaduría } \\
\text { Pública }\end{array}$ & $\begin{array}{l}\text { Enfoques Modernos del Costo; Enfoques } \\
\text { Tradicionales del Costo; Presupuestos }\end{array}$ & $\begin{array}{l}\text { Costos Estratégicos; } \\
\text { Costos Logísticos; } \\
\text { Gerencia del Valor; } \\
\text { Principios de } \\
\text { Manufactura; Teoría de } \\
\text { las Restricciones }\end{array}$ \\
\hline $\begin{array}{l}\text { Universidad } \\
\text { Externado de } \\
\text { Colombia }\end{array}$ & $\begin{array}{l}\text { Contaduría } \\
\text { Pública }\end{array}$ & $\begin{array}{l}\text { Ciclo Contable de la Producción, } \\
\text { Administración de la producción; } \\
\text { Contabilidad Gerencial de la Producción; } \\
\text { Control Gerencial; Presupuestos; } \\
\text { Simulación Gerencial }\end{array}$ & N/A \\
\hline $\begin{array}{l}\text { Universidad } \\
\text { ICESI }\end{array}$ & $\begin{array}{l}\text { Contaduría } \\
\text { Pública y Finanzas } \\
\text { Internacionales }\end{array}$ & $\begin{array}{l}\text { Gerencia de Costos; Presupuestos; } \\
\text { Sistemas y Análisis de Costos }\end{array}$ & $\mathrm{N} / \mathrm{A}$ \\
\hline $\begin{array}{l}\text { Universidad } \\
\text { Libre (Cali) }\end{array}$ & $\begin{array}{l}\text { Contaduría } \\
\text { Pública }\end{array}$ & $\begin{array}{l}\text { Costos Gerenciales; Presupuestos } \\
\text { Empresariales; Simuladores Gerenciales; } \\
\text { Sistemas de Costeo }\end{array}$ & $\begin{array}{l}\text { Gerencia Estratégica de } \\
\text { Costos }\end{array}$ \\
\hline $\begin{array}{l}\text { Universidad } \\
\text { Santo Tomás } \\
\text { (Bogotá) }\end{array}$ & $\begin{array}{l}\text { Contaduría } \\
\text { Pública }\end{array}$ & $\begin{array}{l}\text { Presupuestos: Elaboración y Control; } \\
\text { Sistemas III (Control de Gestión); Teoría de } \\
\text { Costos I; Teoría de Costos II }\end{array}$ & $\mathrm{N} / \mathrm{A}$ \\
\hline
\end{tabular}

Fuente: elaboración propia

\section{La Formación en Contabilidad de Gestión de algunos pregrados de Ciencias Contables en Brasil}

Brasil es uno de los países latinoamericanos con mayor desarrollo en Contabilidad de Gestión, tal como lo proponen las investigaciones de Salgado-Castillo (2011) y João Lunkes et al. (2013). Es probable que la formación de posgrado, a nivel de maestrías y doctorados, contribuya a esta situación. Según la información del Censo de Educación Superior del año 2014, en Brasil existen 1256 programas de Ciencias Contables con registro activo, ofertados por 953 Instituciones de Educación Superior, el 10,7\% son entidades públicas y el $89,3 \%$ son privadas (Instituto Nacional de Estudos e Pesquisas Educacionais Anísio Teixeira - INEP, 2015); siete (7) de esas instituciones se encuentran en el Top 25 de Universidades Latinoamericanas, según el
Ranking QS 2015 de Latinoamérica, los programas ofertados por esas instituciones fueron incluidos en esta investigación.

Al analizar la formación en Contabilidad de Gestión que se oferta en los pregrados en Ciencias Contables, se encuentra que en promedio el 9,12\% de los créditos obligatorios del plan de estudios y el $4,55 \%$ de los créditos optativos se dedica a esta área de formación, tal como se evidencia en la Figura 2.

En los pregrados de Brasil, la formación en Contabilidad de Gestión contempla asignaturas como contabilidad de costos y análisis de costos, en estas se estudian los fundamentos de costos, los sistemas de costeo, el análisis de variaciones, así como metodologías de costeo que se utilizan para la toma de decisiones, como el costeo 
directo, el costeo total, los modelos de análisis costo-volumen-utilidad, entre otros. Es común encontrar la asignatura Contraloría, poco usual en los otros países, al revisar el microcurrículo de las asignaturas de esta temática, se encuentran temas relacionados con el control de gestión y medición del desempeño. La oferta de asignaturas electivas u optativas es más amplia, si se compara con la formación en Colombia, el $85,7 \%$ de los programas analizados tienen optativas en esta área. La Tabla 3 resume las asignaturas obligatorias y optativas, de los programas analizados.

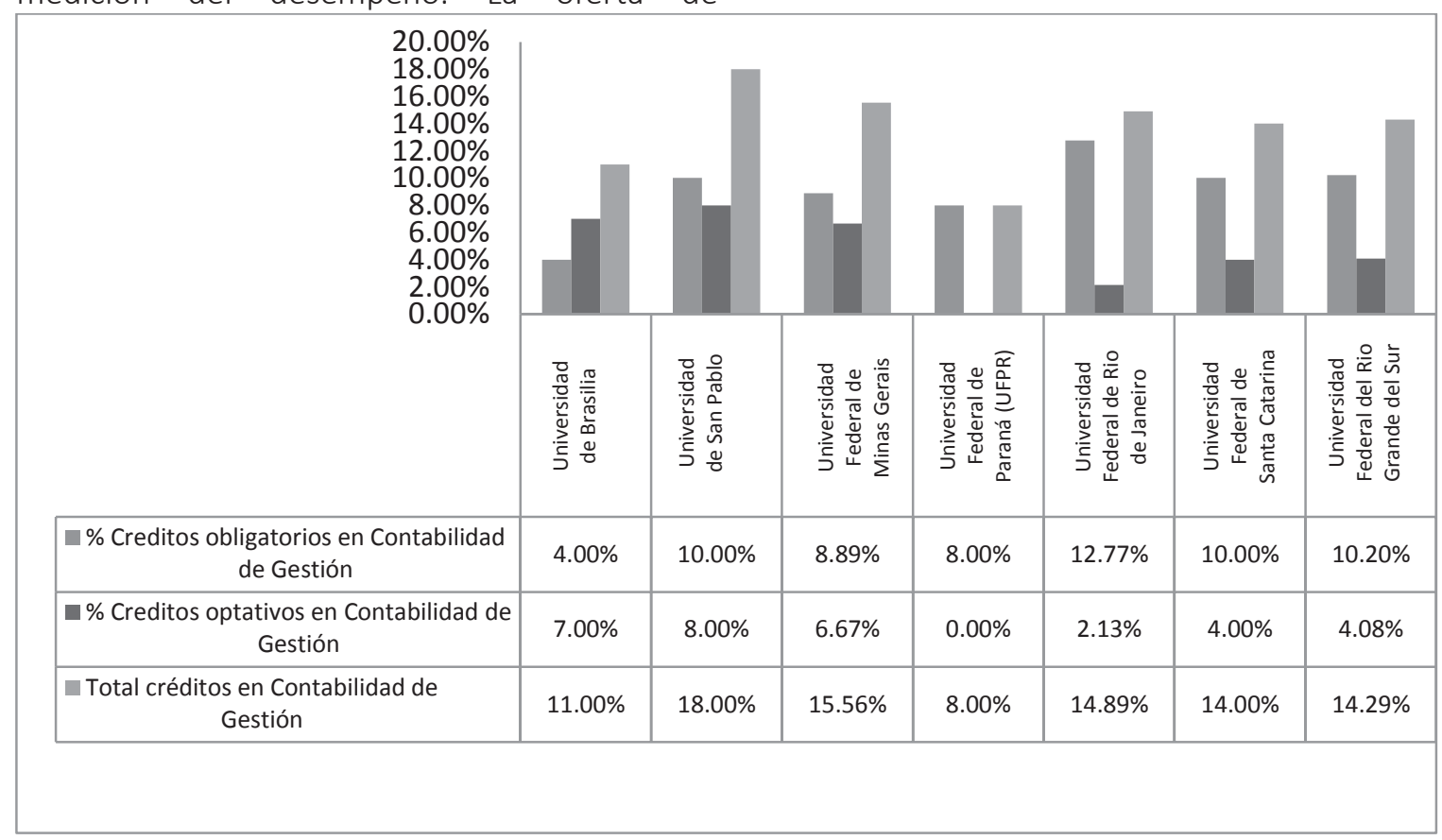

Figura 2. Porcentaje de créditos obligatorios y optativos dedicados a contabilidad de gestión en programas de pregrado en ciencias contables de Brasil.

Figure 2. Percentage of mandatory and electives credits dedicated to management accounting in Brazilian Accounting Undergraduate Programs.

Fuente: elaboración propia.

Tabla 3. Asignaturas obligatorias y optativas en Contabilidad de Gestión de programas de pregrado en ciencias contables de Brasil

Table 3. Mandatory and electives courses in Management Accounting in Brazilian Accounting Undergraduate Programs

\begin{tabular}{|l|l|l|l|}
\hline \multicolumn{1}{|c|}{ Institución } & \multicolumn{1}{|c|}{ Pregrado } & \multicolumn{1}{|c|}{ Asignaturas Obligatorias } & \multicolumn{1}{c|}{ Asignaturas Optativas (Electivas) } \\
\hline $\begin{array}{l}\text { Universidad de } \\
\text { Brasilia }\end{array}$ & $\begin{array}{l}\text { Ciencias } \\
\text { contables }\end{array}$ & Contraloría; Costos & $\begin{array}{l}\text { Costos aplicados al sector público; } \\
\text { Juegos Contables de Empresas; Métodos } \\
\text { y modelos cuantitativos de decisiones; } \\
\text { Presupuesto Empresarial; Teoría de } \\
\text { Contabilidad Gerencial }\end{array}$ \\
\hline $\begin{array}{l}\text { Universidad de } \\
\text { San Pablo }\end{array}$ & $\begin{array}{l}\text { Ciencias } \\
\text { contables }\end{array}$ & $\begin{array}{l}\text { Análisis de costos; Contabilidad de } \\
\text { Costos; Contraloría; Juegos de Empresas } \\
\text { I; Juegos de empresas II; Planeación } \\
\text { estratégica y presupuesto empresarial }\end{array}$ & $\begin{array}{l}\text { Gestión Estratégica de Costos; Solución } \\
\text { de problemas en control gerencial; } \\
\text { Teoría de Restricciones; Toma de } \\
\text { decisiones en contabilidad; Tópicos de } \\
\text { Contabilidad Gerencial; Contraloría en } \\
\text { logística; Contraloría en la Gestión } \\
\text { Pública }\end{array}$ \\
\hline
\end{tabular}




\begin{tabular}{|l|l|l|l|}
\hline \multicolumn{1}{|c|}{ Institución } & \multicolumn{1}{c|}{ Pregrado } & \multicolumn{1}{|c|}{ Asignaturas Obligatorias } & \multicolumn{1}{c|}{ Asignaturas Optativas (Electivas) } \\
\hline $\begin{array}{l}\text { Universidad } \\
\text { Federal de Minas } \\
\text { Gerais }\end{array}$ & $\begin{array}{l}\text { Ciencias } \\
\text { contables }\end{array}$ & $\begin{array}{l}\text { Análisis de costos; Contabilidad de } \\
\text { Costos; Contabilidad Gerencial; } \\
\text { Presupuesto Empresarial }\end{array}$ & $\begin{array}{l}\text { Administración de la producción y de } \\
\text { operaciones; Planeación y Presupuesto } \\
\text { de Instituciones Públicas; Finanzas y } \\
\text { Posicionamiento Estratégico }\end{array}$ \\
\hline $\begin{array}{l}\text { Universidad } \\
\text { Federal de } \\
\text { Paraná (UFPR) }\end{array}$ & $\begin{array}{l}\text { Ciencias } \\
\text { contables }\end{array}$ & $\begin{array}{l}\text { Contabilidad de Costos; análisis de } \\
\text { Costos; Contraloría; Planeación } \\
\text { Estratégica y Presupuesto }\end{array}$ & N/A \\
\hline $\begin{array}{l}\text { Universidad } \\
\text { Federal de Rio de } \\
\text { Janeiro }\end{array}$ & $\begin{array}{l}\text { Ciencias } \\
\text { contables } \\
\text { Contabilidad de Costos I; Contabilidad de Contabilidad Gerencial; } \\
\text { Contraloría; Presupuesto y planeación } \\
\text { empresarial; Presupuesto } \\
\text { Gubernamental }\end{array}$ & Simulación Empresarial \\
\hline $\begin{array}{l}\text { Universidad } \\
\text { Federal de Santa } \\
\text { Catarina }\end{array}$ & $\begin{array}{l}\text { Ciencias } \\
\text { contables }\end{array}$ & $\begin{array}{l}\text { Análisis de Costos; Contabilidad de } \\
\text { Costos; Contabilidad Gerencial; } \\
\text { Contraloría; Simulación gerencial I; } \\
\text { Simulación gerencial II }\end{array}$ & $\begin{array}{l}\text { Tópicos Especiales en Contabilidad } \\
\text { Gerencial I; Tópicos Especiales en } \\
\text { Contabilidad Gerencial II; Tópicos } \\
\text { Especiales en Contabilidad Gerencial III }\end{array}$ \\
\hline $\begin{array}{l}\text { Universidad } \\
\text { Federal del Rio } \\
\text { Grande del Sur } \\
\text { (UFRGS) }\end{array}$ & $\begin{array}{l}\text { Ciencias } \\
\text { contables }\end{array}$ & $\begin{array}{l}\text { Análisis de costos; Contabilidad de } \\
\text { costos I; Contabilidad de costos II; } \\
\text { Contraloría; Organización de la } \\
\text { producción }\end{array}$ & $\begin{array}{l}\text { Planeación y Control de la Producción; } \\
\text { Presupuesto Público }\end{array}$ \\
\hline
\end{tabular}

$$
\text { Fuente: elaboración propia }
$$

\section{La Formación en Contabilidad de Gestión de algunos pregrados de Finanzas y Contabilidad en España}

En Iberoamérica, España sobresale por la producción académica e investigativa en contabilidad de gestión, según la investigación de Salgado-Castillo (2011) y João Lunkes et al. (2013). No obstante, en ese país, el pregrado en Finanzas y Contabilidad es relativamente reciente, surge con posterioridad al año 2000, en el proceso de reforma universitaria emprendido por el Ministerio de Educación de España (Arquero-Montaño, Donoso-Anes, JiménezCardoso, y González González, 2009). Antes, las labores contables eran ejercidas por licenciados en ciencias empresariales o dirección de empresas.

En España existen 28 programas de grado en Finanzas y Contabilidad, ofertados por 23 Instituciones de Educación Superior, en su mayoría (95,65\%) de carácter público (España Ministerio de Educación Cultura y Deporte, 2015), de las cuales siete (7) se encuentran en el
Ranking QS de Universidades Europeas, estas fueron incluidas en esta investigación.

Al analizar el número de créditos dedicados a la formación en Contabilidad de Gestión, en algunos pregrados de Finanzas y Contabilidad españoles, se encuentra que en promedio el $3,75 \%$ y el $3,87 \%$ de los créditos del plan de estudio corresponden a asignaturas obligatorias y optativas, respectivamente, en esta área de conocimiento, así se evidencia en la Figura 3.

En las asignaturas de Contabilidad de Gestión que se consideran obligatorias, se aborda el estudio de los fundamentos de costos, los sistemas de costeo y el análisis de costos, principalmente. En las asignaturas optativas se incorporan temáticas relacionadas con la toma de decisiones, especialmente métodos cuantitativos que apoyan la toma de decisiones y la gestión organizacional. En la Tabla 4 se presenta una síntesis de las asignaturas obligatorias y optativas que se ofertan en los pregrados estudiados. 


\begin{tabular}{|c|c|c|c|c|c|c|c|c|}
\hline \multicolumn{9}{|c|}{$12.00 \%$} \\
\hline \multicolumn{9}{|c|}{$10.00 \%$} \\
\hline \multicolumn{9}{|c|}{$8.00 \%$} \\
\hline \multicolumn{9}{|c|}{$6.00 \%$} \\
\hline \multicolumn{9}{|c|}{$4.00 \%$} \\
\hline \multirow{2}{*}{\multicolumn{2}{|c|}{ 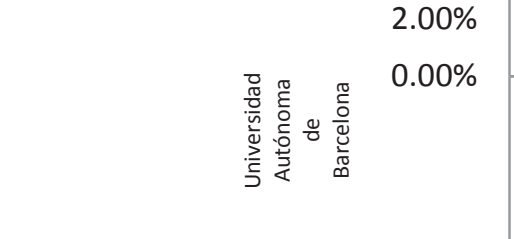 }} & & & & & & & \\
\hline & & 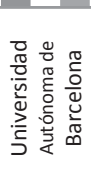 & 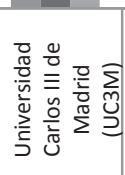 & 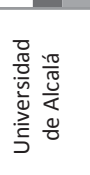 & 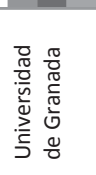 & 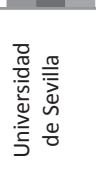 & 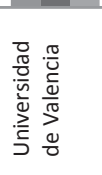 & 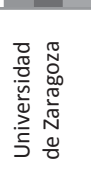 \\
\hline \multicolumn{2}{|c|}{$\begin{array}{c}\text { \% Creditos obligatorios en Contabilidad } \\
\text { de Gestión }\end{array}$} & $5.00 \%$ & $5.00 \%$ & $0.00 \%$ & $5.00 \%$ & $5.00 \%$ & $3.75 \%$ & $2.50 \%$ \\
\hline \multicolumn{2}{|c|}{$\begin{array}{c}\text { \% Creditos optativos en Contabilidad de } \\
\text { Gestión }\end{array}$} & $0.00 \%$ & $5.00 \%$ & $8.13 \%$ & $2.50 \%$ & $5.00 \%$ & $1.88 \%$ & $4.58 \%$ \\
\hline \multicolumn{2}{|c|}{ Total créditos en Contabilidad de Gestión } & $5.00 \%$ & $10.00 \%$ & $8.13 \%$ & $7.50 \%$ & $10.00 \%$ & $5.63 \%$ & $7.08 \%$ \\
\hline
\end{tabular}

Figura 3. Porcentaje de créditos obligatorios y optativos dedicados a contabilidad de gestión en programas de pregrado en finanzas y contabilidad de España

Figure 3. Percentage of mandatory and electives credits dedicated to management accounting in Spanish Accounting Undergraduate Programs

Fuente: elaboración propia

Tabla 4. Asignaturas obligatorias y optativas en Contabilidad de Gestión de programas de pregrado en Finanzas y Contabilidad de España

Table 4. Mandatory and electives courses in Management Accounting in Spanish Accounting Undergraduate Programs

\begin{tabular}{|c|c|c|c|}
\hline Institución & Pregrado & Asignaturas Obligatorias & Asignaturas Optativas (Electivas) \\
\hline $\begin{array}{l}\text { Universidad Autónoma } \\
\text { de Barcelona }\end{array}$ & $\begin{array}{l}\text { Contabilidad } \\
\text { y Finanzas }\end{array}$ & $\begin{array}{l}\text { Contabilidad de Costos I; } \\
\text { Contabilidad de Costos II }\end{array}$ & N/A \\
\hline $\begin{array}{l}\text { Universidad Carlos III de } \\
\text { Madrid (UC3M) }\end{array}$ & $\begin{array}{l}\text { Finanzas y } \\
\text { Contabilidad }\end{array}$ & $\begin{array}{l}\text { Control de Gestión; } \\
\text { Gestión de Costos }\end{array}$ & $\begin{array}{l}\text { Contabilidad para la alta dirección; } \\
\text { Optimización y simulación en la } \\
\text { empresa }\end{array}$ \\
\hline Universidad de Alcalá & $\begin{array}{l}\text { Finanzas y } \\
\text { Contabilidad }\end{array}$ & $\mathrm{N} / \mathrm{A}$ & $\begin{array}{l}\text { Contabilidad de costos; Contabilidad } \\
\text { de Gestión; Econometría aplicada a } \\
\text { toma de decisiones }\end{array}$ \\
\hline Universidad de Granada & $\begin{array}{l}\text { Finanzas y } \\
\text { Contabilidad }\end{array}$ & $\begin{array}{l}\text { Contabilidad de Gestión I; } \\
\text { Contabilidad de Gestión II }\end{array}$ & Contabilidad de Gestión Avanzada \\
\hline Universidad de Sevilla & $\begin{array}{l}\text { Finanzas y } \\
\text { Contabilidad }\end{array}$ & $\begin{array}{l}\text { Contabilidad de Costos; } \\
\text { Contabilidad para la } \\
\text { gestión }\end{array}$ & $\begin{array}{l}\text { Control de Gestión; Simulación } \\
\text { Empresarial }\end{array}$ \\
\hline Universidad de Valencia & $\begin{array}{l}\text { Finanzas y } \\
\text { Contabilidad }\end{array}$ & Contabilidad de Gestión & Control Presupuestario y de Gestión \\
\hline Universidad de Zaragoza & $\begin{array}{l}\text { Finanzas y } \\
\text { Contabilidad }\end{array}$ & Contabilidad Analítica & $\begin{array}{l}\text { Gestión de procesos productivos; } \\
\text { Sistemas decisionales }\end{array}$ \\
\hline
\end{tabular}

Fuente: elaboración propia 


\section{CONCLUSIONES Y REFLEXIONES FINALES}

El propósito de este artículo fue explorar la formación en Contabilidad de Gestión, presente en los currículos de algunos pregrados de Contaduría de Colombia, Brasil y España, con el propósito de identificar las temáticas que se abordan y la naturaleza obligatoria u optativa que se le otorga en la formación. Los resultados indican que las temáticas que se estudian en los tres países son similares, pero varía el número de créditos académicos y la obligatoriedad u optatividad de los mismos. En los pregrados de Contaduría colombianos analizados, se dedica en promedio el 8,28\% de los créditos a la formación obligatoria en Contabilidad de Gestión, cercano a lo que se dedica en Brasil $(9,12 \%)$ y el doble de lo que se exige en los pregrados de España $(3,75 \%)$. Algunos pregrados colombianos $(42,8 \%)$ ofertan líneas optativas o asignaturas electivas en esta área; esto contrasta con la formación que se imparte en Brasil y España, países en los cuales, el $85,7 \%$ de los programas estudiados, tienen optativas o líneas de profundización en Contabilidad de Gestión. No obstante, es preciso reconocer las limitaciones de esta investigación basada en fuentes documentales - planes de estudio y microcurrículos-, estas permiten comprender el «deber ser» o lo que se espera que se aborde en los procesos de formación en Contabilidad de Gestión, según lo declara el programa de cada institución analizada, pero pueden existir variaciones en la práctica formativa en cuanto a los temas que se enseñan y en las estrategias pedagógicas, que pueden conducir a resultados diferentes a los declarados en los programas, futuras investigaciones pueden centrar su atención en estos aspectos.

Para fortalecer la formación en Contabilidad de Gestión no es suficiente con incrementar el número de asignaturas o de créditos del currículo destinados a esta área, también es necesario fomentar entre los docentes y los estudiantes el espíritu investigativo en este campo de conocimiento, avanzar en el desarrollo de posgrados -especializaciones, maestrías y doctorados - para la transferencia y la generación de conocimientos, así como la constitución de redes y espacios académicos revistas profesionales y científicas, seminarios, congresos - para la construcción colectiva y la difusión de conocimiento en contabilidad de gestión. Quizá esa es una de las razones por las cuales la formación y la investigación en Contabilidad de Gestión en España y Brasil han alcanzado un mayor posicionamiento $y$ visibilidad en Iberoamérica, en comparación con la situación de Colombia, donde la disciplina se encuentra en un estadio incipiente (SalgadoCastillo, 2011), (Duque Roldán y Osorio Agudelo, 2013). La formación de alto nivel que se imparte en los doctorados es fundamental para el desarrollo investigativo de los países, por mencionar un dato que ilustra las diferencias en los países estudiados, en Brasil existen 13 doctorados en Contabilidad, en España 8 y en Colombia no hay ninguno propio, según datos del SNIES a noviembre de 2015. Adicionalmente, la conformación de grupos de estudio, semilleros y grupos de investigación, con líneas de trabajo en contabilidad de gestión, es fundamental para promover, tanto en la academia como en la práctica organizacional, la incursión de los contadores en la contabilidad de gestión. Sin embargo, es necesario reconocer que la confidencialidad de la información de la contabilidad de gestión (Moehrle et al., 2009, p. 436) hace más difícil incursionar en este campo, tanto para las prácticas académicas de los estudiantes como para quienes se interesan en la investigación.

Es importante que desde la formación de pregrado se explique a los estudiantes de Contaduría las diferentes posibilidades de ejercicio profesional, estas no se limitan a la contabilidad financiera y la contabilidad tributaria, que han sido las áreas con mayor tradición, sino que existen otras opciones con un gran potencial en campos como la contabilidad de gestión (Fregona, 2007, p. 84). En este sentido, los docentes de esta área de 
conocimiento juegan un rol fundamental (LeónPaime y Ardila-Trujillo, 2013), son ellos los principales motivadores, para que los estudiantes puedan comprender el rol estratégico que el contador puede llegar a desempeñar en las organizaciones, como un profesional que agrega valor y esto es posible desde la contabilidad de gestión.

Desde los procesos de formación es necesario comprender y abordar la interdisciplinariedad de la contabilidad de gestión (Salgado-Castillo, 2014, p. 800), pues la participación en esta área de conocimiento y de práctica requiere de conocimientos y habilidades que se desarrollan desde diversos campos como las matemáticas y la estadística, las ciencias económicas, el derecho, la administración y la ética. Las matemáticas y la estadística ayudan a desarrollar el razonamiento lógico y proveen la fundamentación y las herramientas cuantitativas para el análisis y la modelación de la información de costos, indispensables para la planeación, el control y la toma de decisiones. Las ciencias económicas permiten comprender el contexto en el que se desenvuelven las organizaciones y la forma como algunas variables - por ejemplo, oferta, demanda-, afectan los costos y los precios. El derecho, en sus múltiples especialidades -laboral, comercial, tributario, entre otros - ayuda a comprender los marcos jurídicos que regulan las transacciones y los acuerdos que realizan o planean realizar las empresas, y de los cuales da cuenta la contabilidad de gestión, a través del registro y el análisis de los costos y la elaboración de los presupuestos. Del mismo modo, la formación en administración es fundamental en contabilidad de gestión, porque permite comprender cómo se articulan los procesos y las funciones que se realizan en una organización y las herramientas que se pueden implementar para mejorar la eficiencia y la eficacia del sistema organizacional - por ejemplo, la gestión por procesos, la cadena de valor-, algunas de ellas son indispensables para comprender y aplicar los desarrollos contemporáneos de la contabilidad de gestión, como los sistemas de costos $A B C$, por ejemplo. Asimismo, la ética es un componente indispensable en la formación y en la práctica profesional, la búsqueda de la eficiencia y la eficacia no puede ir en contra de los derechos, el respeto y la dignidad que le asisten a cada ser humano, ni en detrimento de las condiciones del entorno; por otra parte, se trabaja con información para la toma de decisiones de los grupos de interés, lo cual exige transparencia y veracidad en los datos suministrados.

Del mismo modo, es importante mejorar la formación en sistemas de información, porque estos son indispensables para el registro y el análisis de la información que genera la contabilidad de gestión. Cabe destacar, asimismo, que los contadores no solo deben ser competentes en utilizar los sistemas de información, sino que además deben estar preparados para participar con otros profesionales en el diseño, gestión y control de los mismos (International Accounting Education Standards Board - IAESB, 2014, p. 39). Esta situación es más relevante en el contexto colombiano, pues, la investigación de BarretoCarvajal, Cárdenas-Mora, y MondragónHernández (2011, p. 268), indica que en la formación de contadores no es frecuente el uso sistemas de información para el desarrollo de competencias relacionadas con la preparación, la presentación y el control de información. Por otra parte, los sistemas de información integrados que poseen módulos para la gestión de costos son escasos y de alto costo, esto los hace prácticamente inasequibles para las micro, pequeñas y medianas empresas que representan el $99 \%$ del total de las empresas en Colombia (Dinero, 2015) y América Latina (OCDE y CEPAL, 2012). Algunas veces, esta dificultad se supera con desarrollos internos -módulos o programas informáticos-que realizan los ingenieros con el apoyo de contadores, para suplir necesidades específicas de una empresa; en otros casos, el contador se debe ingeniar la manera de organizar y procesar la información por sus propios medios, por esta razón es importante fortalecer la formación en aspectos tales como lógica de programación, bases de datos, asuntos 
que pueden ser incluidos como asignaturas optativas, como lo ofertan algunos pregrados de Brasil.

Por otra parte, en contabilidad de gestión no solo interesa el registro y el análisis de la información de costos, también es necesaria la simulación y modelación de esta información para los procesos de planeación y toma de decisiones. Por lo tanto, es necesario fortalecer la formación en métodos y modelos cuantitativos que apoyen estos aspectos. En varios programas españoles analizados y en algunos de Brasil, ya se incluyen estos aspectos, pero no se evidencia en los programas colombianos que fueron estudiados en esta investigación.

En síntesis, la formación en contabilidad de gestión como parte de los currículos de programas de Contaduría, se constituye en un escenario propicio para la mirada holística y la integración de conocimientos de diferentes áreas, tan necesarias en la formación de los contadores (Conferencia de las Naciones Unidas sobre Comercio y Desarrollo -UNCTAD, 2003, p. 68) Es así como, algunas universidades comienzan a incluir asignaturas como simulación gerencial, juego de empresas, que en esta investigación se consideraron parte de la contabilidad de gestión. Otra opción, es utilizar estrategias, como el estudio de casos y el aprendizaje basado en problemas, para estudiar temas específicos de esa área, así se evidenció en algunas asignaturas de la Universidad de San Pablo, en Brasil. Futuras investigaciones pueden enfocarse en estudiar, por ejemplo, las metodologías de enseñanza aprendizaje que se implementan en los cursos de Contabilidad de Gestión, para identificar aquellas que pueden ser apropiadas para promover y mejorar la formación en esta área.

\section{REFERENCIAS}

Aguiar, H.; Cadavid, L. A.; Cardona, J.; Carvalho, J.; Jiménez, J. y Upegui, M. E. (1998). Diccionario de términos contables para Colombia (Segunda ed.). Medellín: Editorial Universidad de Antioquia.

Ahadiat, N. (2008). In Search of Practice-Based Topics for Management Accounting Education. Management Accounting Quarterly, 9(4), 42-54.

Amat S,, O.; Amat S., J. M. y Álvarez L., J. A. (1996). Contabilidad de Gestión Avanzada: planificiación, control y experiencias prácticas. Madrid: McGraw-Hill.

Arquero M., J. L.; Donoso A., J. A.; Jiménez C., S. M. y González G., J. M. (2009). Análisis exploratorio del perfil demandado para administración y dirección de empresas: implicaciones para el área contable. Revista de Contabilidad - Spanish Accounting Review, 12(2), 181-213.

Barreto C., Ó. M.; Cárdenas M., S. M. y Mondragón H., S. A. (2011). Las tecnologías de información y comunicación en la fomación de contadores públicos: análisis de uso y aplicaciones en cinco universidades colombianas. Cuadernos de Contabilidad, 12(30), 243-272.

Caldera, J.; Baujín, P.; Ripoll F., V. M. y Vega, V. (2007). Evolución en la configuración de los sistemas de costeo basado en las actividades. Actualidad Contable FACES, 10(14), 13-28.

Calleja B., F. J. y Calleja, F. (2014). Contabilidad Administrativa. Contabilidad Gerencial. Segunda edición. México: Pearson Educación.

Conferencia de las Naciones Unidas sobre Comercio y Desarrollo -UNCTAD. (2003). Modelo revisado de plan de estudios de contabilidad. Ginebra: Grupo de Trabajo Intergubernamental de Expertos en Normas Internacionales de Contabilidad.

Dinero (2015). ¿¿Por qué fracasan las pymes en Colombia? Recuperado de www.dinero.com 
Duque R., M. I. y Osorio A., J. A. (2013). ¿El proceso de convergencia en Colombia excluye la contabilidad de costos? Cuadernos de Contabilidad, 14, 11211146.

Duque R., M. I. y Osorio A., J. A. (2013). Estado actual de la investigación en Costos y Contabilidad de Gestión en Colombia. Revista del Instituto Internacional de Costos, (11), 26-42.

Escobar B., J. (1991). Fundamentos de Contabilidad Gerencial. Medellín: Universidad Eafit.

Escobar R., T. y Cortijo G., V. (2012). Fundamentos de contabilidad de gestión. Madrid: Pearson Educación.

España - Ministerio de Educación Cultura y Deporte (2015). Oferta de titulaciones.

Evia P., M. (2006). ¿Es la contabilidad administrativa una herramienta útil para desarrollar la competitividad de las empresas? Contaduría y Administración, (219), 145-166.

Fregona, R. (2007). El plan de estudio contable y las demandas de la actualidad. Revista del Instituto Internacional de Costos, (1), 60-87.

Frezatti, F.; De Aguiar, A. B. y Guerreiro, R. (2007). Diferenciações Entre A Contabilidade Financeira E A Contabilidade Gerencial: Uma Pesquisa Empírica A Partir De Pesquisadores De Vários Países. Revista Contabilidade \& Financas-USP, (44), 9-22.

Gómez, M. (2007). Comprendiendo las relaciones entre los sistemas contables, los modelos contables y los sistemas de información contables empresariales. Revista Internacional Legis de Contabilidad \& Auditoría, (32), 235-253.

González, P. (2010). Avances y desafíos sobre la enseñanza de la contabilidad de gestión en Colombia: desde una perspectiva de Mipymes y de la educación superior.
Revista Universo Contabil, 6(3), 140-155. doi: 10.4270/ruc.2010327

Harris, J. y Durden, C. (2012). Management Accounting Research: An Analysis of Recent Themes and Directions for the Future. Journal of Applied Management Accounting Research, 10(2), 21-41.

Hesford, J.; Lee, S. H. y Van Der Stede, W. (2007). Management accounting: a bibliographic study. In C. Chapman, A. Hopwood \& M. Shields (Eds.), Handbook of management accounting research (pp. 3-26). Oxford, United Kindgon: Elsevier.

Instituto Nacional de Estudos e Pesquisas Educacionais Anísio Teixeira - INEP (2015). Censo da Educação Superior 2014 - Brasil. Brasil: INEP.

International Accounting Education Standards Board - IAESB (2014). Handbook of International Education Pronouncements. New York: International Federation of Accountants - IFAC,

Jiles, L. (2014). Management Accounting Career Readiness: Shaping Your Curriculum. Strategic Finance, 96(8), 3842.

João Lunkes, R.; Ripoll Feliu, V. y Silva da Rosa, F. (2012). Análise quantitativa da producao e da formacao de doutores em contabilidadde gerencial: um estudo no cenario espanhol. Revista Universo Contabábil, 8(2), 118-133.

João Lunkes, R.; Ripoll Feliu, V. M. y Silva da Rosa, F. (2013). Study of Published Articles on Management Accounting in Brazil and Spain. Revista Contabilidade \& Finanças USP, 24(61), 11-26.

Johnson, T. y Kaplan, R. S. (1988). La Contabilidad de Costes. Auge y caída de la contabilidad de gestión. Barcelona: Plaza \& Janes.

León-Paime, E. F. y Ardila T., M. E. (2013). La práctica docente en contabilidad de gestión; una aproximación a partir de las 
trayectorias. . Cuadernos de Contabilidad, 14(35), 617-637.

Loaiza R., F.; Cárdenas M., S. M. y Peralta B., D. A. (2014). Aproximación a la relación control de gestión-contabilidad administrativa. Gestión \& Sociedad, 7(1), 143-156.

Moehrle, S. R.; Anderson, K. L.; Ayres, F. L.; Bolt-Lee, C. E.; Debreceny, R. S., Dugan, M. T.; Plummer, E. (2009). The Impact of Academic Accounting Research on Professional Practice:An Analysis by the AAA Research Impact Task Force. Accounting Horizons, 23(4), 411-456.

OCDE, \& CEPAL (2012). Perspectivas Económicas de América Latina 2013: Políticas de Pymes para el Cambio Estructural.

Osorio, O. (1991). La capacidad de producción y los costos. Segunda edición. Buenos Aires: Ediciones Macchi.

Ramírez P., D. N. (2013). Contabilidad administrativa: un enfoque estratégico para competir. Novena edición. México: McGraw-Hill Interamericana Editores.

Romero C., A. (1997). La contabilidad gerencial y los nuevos métodos de costeo. México: Instituto Mexicano de Contadores Públicos.

Salgado C., J. A. (2011). Tendencias de investigación en contabilidad de gestión en Iberoamérica (1998-2008). Cuadernos de Contabilidad, 12(30), 273-305.
Salgado C., J. A. (2014). Tendencias en contabilidad de gestión: una mirada a su evolución (finales del siglo XIX y siglo XX). Cuadernos de Contabilidad, 15(39), 787805. doi: 10.11144/Javeriana.cc1539.tcgm

Sanabria, M. (2010). De los conceptos de administración, gobierno, gerencia, gestión y management: algunos elementos de corte epistemológico y aportes para una mayor comprensión. Universidad \& Empresa, 9(13), 155-194.

Shank, J. K. y Govindarajan, V. (1998). Gerencia estratégica de costos. La nueva herramienta para desarrollar una ventaja competitiva. Bogotá: Norma.

Siegel, G.; Sorensen, J. E.; Klammer, T. y Richtermeyer, S. B. (2010). The Ongoing Preparation Gap in Management Accounting Education: A Guide for Change. Management Accounting Quarterly, 11(4), 29-39.

Sinisterra, G. y Polanco, L. E. (2007). Contabilidad Administrativa. Bogotá: Ecoe Ediciones.

Torrecilla, A.; Fernández, A. y Gutiérrez, G. (1993). Contabilidad de costes y contabilidad de gestión. Volumen 1 (McGraw-Hill Ed.). Madrid.

Vanegas N., N.; García P. de L., D. y Marín H., S. (2009). Estrategia, sistemas de contabilidad de gestión y rendimiento en la industria de la conserva de frutas y vegetales. Cuadernos de Administración, 22(39), 213-233. 